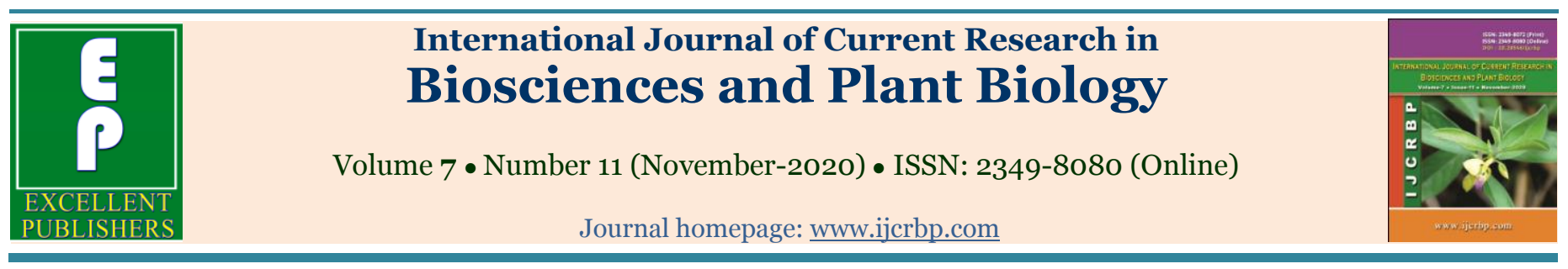

Original Research Article

doi: $\underline{\text { https://doi.org/10.20546/ijcrbp.2020.711.001 }}$

\title{
Birds diversity of the Bouafle Classified Forest (Central-western of Côte d'Ivoire)
}

\section{Gnininté Maxime ZEAN, Dibié Bernard AHON* and Béné Jean-Claude KOFFI}

Laboratory of Biodiversity and Tropical Ecology, University Jean Lorougnon GUEDE, Daloa, Cote d'Ivoire

*Corresponding author; e-mail bahon2013@gmail.com

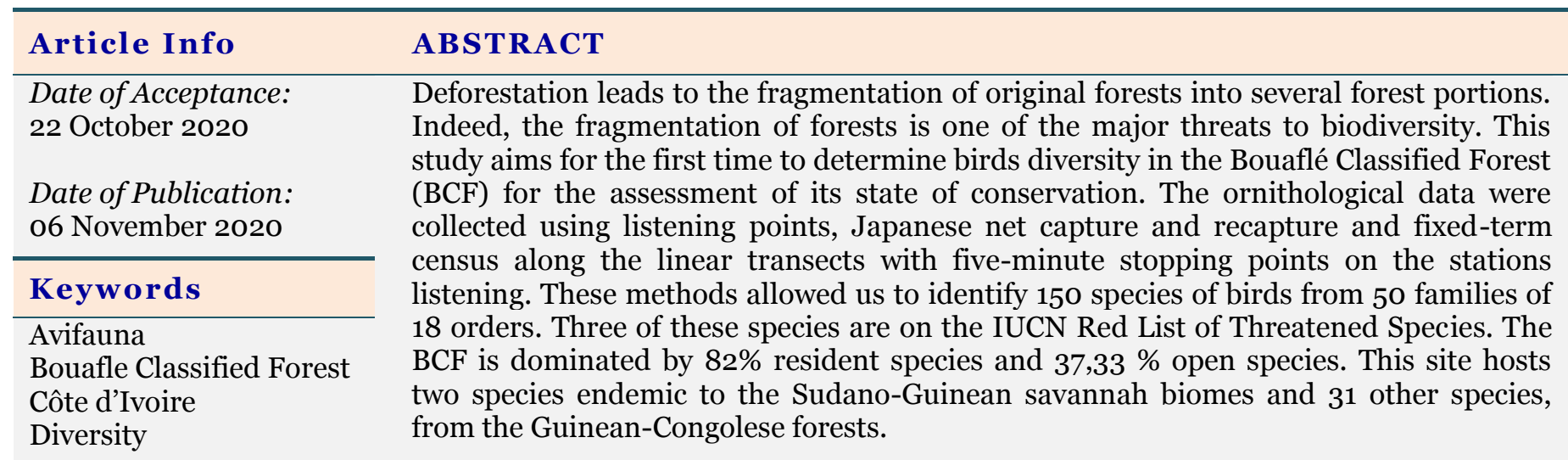

\section{Introduction}

The alteration of natural ecosystems is the greatest threat to biodiversity (Meffe and Caroll, 1994; N'Da et al., 2008; Murhabale et al., 2020). It can lead to the fragmentation of forest habitats in favour of forest fragments (Saunders et al., 1991). This phenomenon can be observed worldwide, especially in tropical forests (Vallan, 1999). Tropical rainforests, which are home to the largest known diversity of plant and animal species (Puig, 2001 ; Teyssèdre, 2004), declined considerably during the 20th century (Lévêque, 1994). In Côte d'Ivoire, biodiversity is characterised by significant richness and diversity (Halle and Bruzon, 2006). However, this developing country is faced with an unprecedented reduction in its forest area
(Mayaux et al., 2003; Brou et al., 2005; Koné et al., 2014) which promises serious consequences both ecologically and economically. This decline in vegetation cover is the result of a development choice based on agriculture (Konan, 2009). Unfortunately, the galloping degradation of the natural environment has led to the disappearance of thousands of hectares of forest with the result that many animal species have become extinct (N'Da et al., 2008).

It is estimated that a few thousand hectares of forests that have not yet been exploited are confined to Classified Forests, Forest Reserves and National Parks (N'Da et al., 2008; Kouakou et al., 2015). Unfortunately, these protected areas are themselves under severe attack, mostly 
through the development of agricultural activities (N'Da et al., 2008; Bitty et al., 2013). This state of affairs is strongly observed in the Bouafle Classified Forest (FCB). Faced with the rather gloomy consequences of deforestation and forest degradation, there is a growing conviction that the situation must no longer be allowed to continue, so we must react.

The collection and analysis of scientific information in a timely manner is essential for interventions in the sustainable management of natural resources, particularly avifauna resources. Moreover, there are several reasons why birds are so often used as a model in the study of ecosystems. Indeed, birds use a wide variety of natural habitats and are therefore directly affected by ecosystem disturbances. They therefore act as bio-indicators of the state of conservation and functioning of ecosystems, landscape modification and climate change (Bibby et al., 1992; Demey and Rainey, 2005; Gottschalk et al., 2007). Their mobility enables them to react instantaneously to any change in the environment. The objective of this study is to determine, for the first time, birds diversity of this Classified Forest in order to assess its conservation status.

\section{Materials and methods}

\section{Materials}

A GPS (Global Positioning System) navigator was used to record geographical coordinates, plot routes and mark the various observation points. The birds were observed using a pair of binoculars (Bushnell, $10 \times 50 \mathrm{~mm}$ ), a headlamp and a torch (Garrity) for lighting the tracks and trails. As for identification, it was carried out using identification guides (Borrow and Demey, 2001). The bird songs are from the Chappuis (2000). These songs were broadcast using a compact disc player (MDM-14 marker and baffles (Sony)). The recording equipment consisted of a digital camera (Panasonic Lumix DMC-TZ 61) for taking pictures, ornithological data sheets to determine the avifaunal population of each study site and a dictaphone (Sony; Cassette; TCM-15O) connected to a micro-directional recorder for recording vocalisations. For the capture and recapture of bird species, two mist nets of $12 \mathrm{~m}(12 \times 2.5 \mathrm{~m}, 16 \mathrm{~mm}$ mesh size) were used.

\section{Methods}

\section{Description of study site}

The BCF is located in the central-western part of Côte d'Ivoire in the mesophilic forests (Guillaumet and Adjanohoun, 1971; Monnier, 1983), about 30 $\mathrm{km}$ from the town of Daloa and $50 \mathrm{~km}$ from the town of Bouaflé. It covers an area of 20,350 ha and is bounded by longitudes $6^{\circ} \mathrm{O} 2^{\prime}$ and $6^{\circ} 13^{\prime}$ West and latitudes $6^{\circ} 42^{\prime}$ and $6^{\circ} 52^{\prime}$ North. Administratively, this forest is located between the regions of Marahoué and Haut-Sassandra, 20\% of its surface belongs to the department of Daloa and 80\% to the department of Bouafle. It is bordered in its northern part by the Daloa-Bouafle road and the Marahoué National Park, to the east and southeast by the Sub-prefecture of Bonon, to the southwest by the Sub-prefectures of Zaguiéta and to the west by the Sub-prefectures of Gadouan and Gonate (Fig. 1). The climate is of the sub-equatorial Attiéen type according to Eldin (1971). Over the year, its average temperature is $25.9^{\circ} \mathrm{C}$. The average annual rainfall is $1301 \mathrm{~mm}$. The vegetation of the FCB belongs to the semi-deciduous dense forest zone (Guillaumet and Adjanohoun, 1971). Some of the biotopes found in the CBF are shown in Fig. 2, while its fauna is not well known because it has not been the subject of any serious study before the present study.

\section{Data collection and analysis}

The study was carried out from February 2019 to May 2020. The BCF was subdivided into three sample sites in addition to the outlying area. This subdivision was made on the basis of the presence or absence of wetlands, the heterogeneity of the vegetation and the degree of anthropisation (the intensity or extent of human activities) of the environment. The methods used are respectively the listening point method (Bibby et al., 1992; Bibby et al., 2000; Yaokokoré-Béibro et al., 2015; Zean et al., 2018), capture and recapture with mist nets (Bennun et al, 2004), time-limited census along line transects (Yaokokoré-Béibro, 2001; Issiaka, 2002 and 2011) with five-minute stopping points at listening stations (Zean et al., 2018). In fact, the fixed listening point method and the mist net capture and recapture method (excluding transects) were used for a whole day between $06: 30$ and 18 : 30 (i.e. $12 \mathrm{~h}$ of daily observation per study site). 


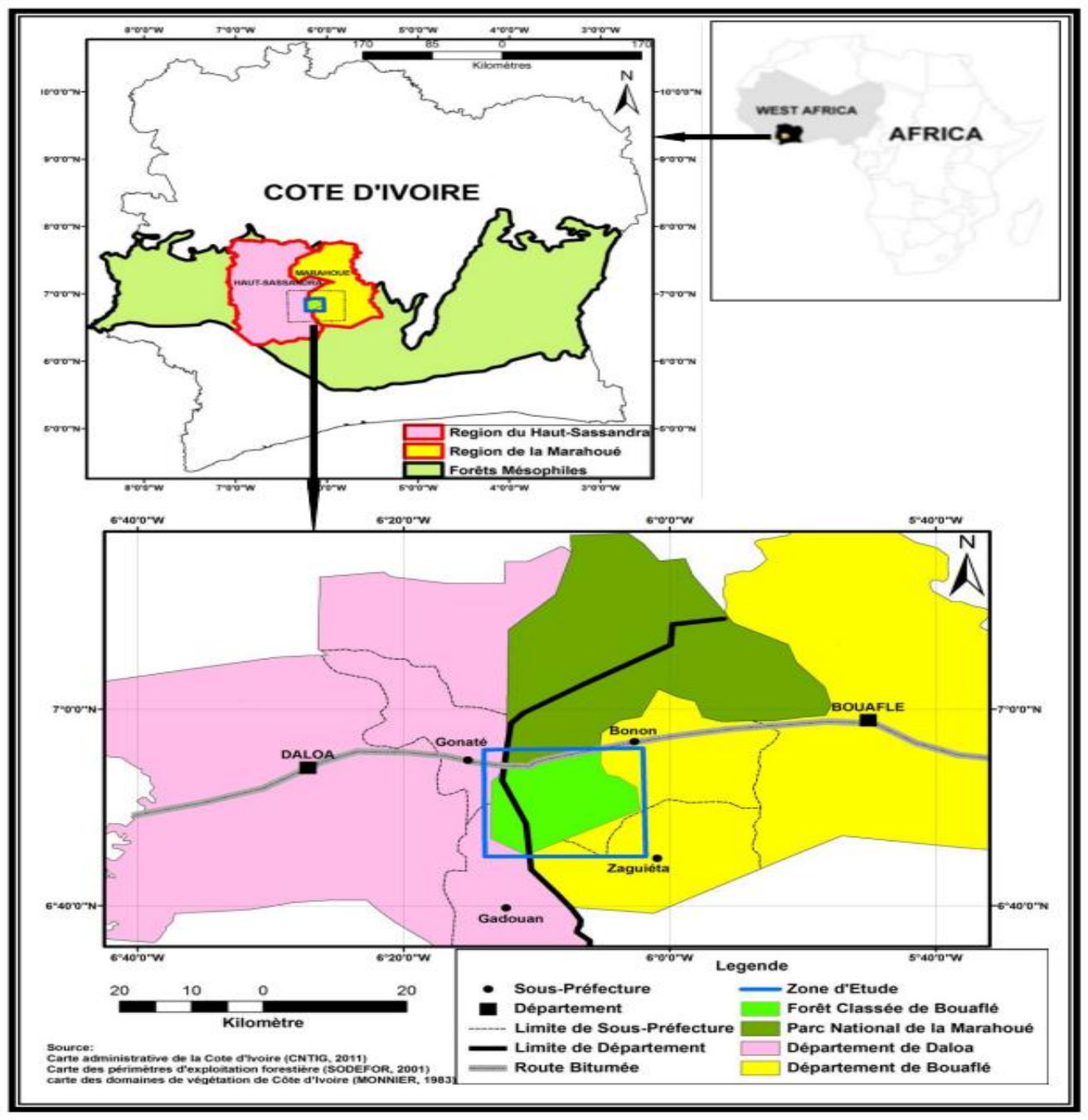

Fig. 1: Map of the location of the BCF in Côte d'Ivoire.

Daytime observations (from $06: 30$ to $10: 30$ in the morning and in the afternoon from $15: 30$ to $18: 30$ ), were carried out on each sample route of each sample site. The inventories were based on a systematic count of all bird species identified, spotted by sight or heard along the four transects of $2 \mathrm{~km}$ each, during a slow ( 0.5 to $1 \mathrm{~km} / \mathrm{h}$ ) and silent walk. Five listening and observation stations, $500 \mathrm{~m}$ apart, were set up on each transect to avoid double counting of individuals during sampling and at least $50 \mathrm{~m}$ from the edges to obtain better results (Delahaye, 2006; Lougbégnon, 2007). These routes were made on the outward and return journey by favourable meteorology (uncovered sky, absence of heavy rain). For nocturnal observations, inventories were carried out on the sample routes used for sampling diurnal species. Three listening stations (1 km apart) were set up on each $1 \mathrm{~km}$ transect. The main methodology was the vocalization replay technique. The songs of all nocturnal bird species such as Nighthawks, some lapwings and birds of prey likely to be encountered in the site were reviewed (10 in total). The birds' songs were broadcast using a compact disc player. The vocalisation of a given species was listened to for 1 minute, followed by a 1-minute wait before moving on to another species. Observations were made during the full moon, from 19:00 to 23:00 on the outward journey and from 04:00 to 06:00 on the return journey. For each of the species listed, the preferred habitat was indicated (FF: highly dependent on forests; F: general forest generalist; F: open habitat; E: forest species; E: forest species; $\mathrm{F}$ : forest species; $\mathrm{E}$ : forest species; $\mathrm{F}$ : forest species; E: forest species; F: forest species; F: forest species; $\mathrm{E}$ : forest species: wetland) and conservation status are from IUCN (2020), the migratory or biogeographical status (resident, 
intra-African migratory or palearctic migratory) according to de Borrow and Demey (2001) and the indications concerning the biomes (GuineanCongolese forest (GC), Sudano-Guinean savannah (SG)) are from Stattersfield et al. (1998) and Fishpool and Evans (2001). It should be noted that in order to standardise the names of the birds recorded in the different tables, reference has been made to the nomenclature, taxonomy and order of Borrow and Demey (2001). The nomenclature, taxonomy and order of species were established according to the Handbook of the Birds of the World and Bird Life International as published by Lepage (2017).

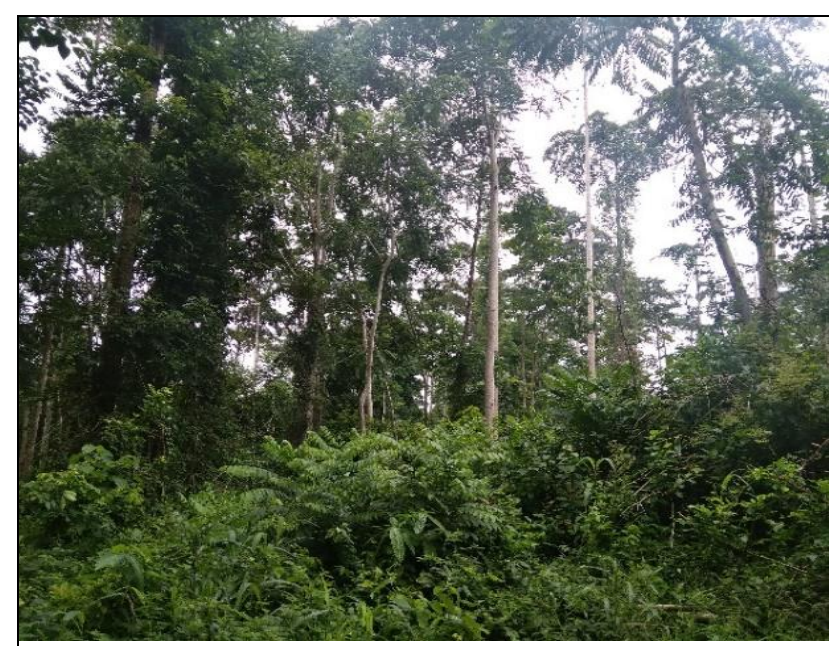

(a) Multispecific woody vegetation

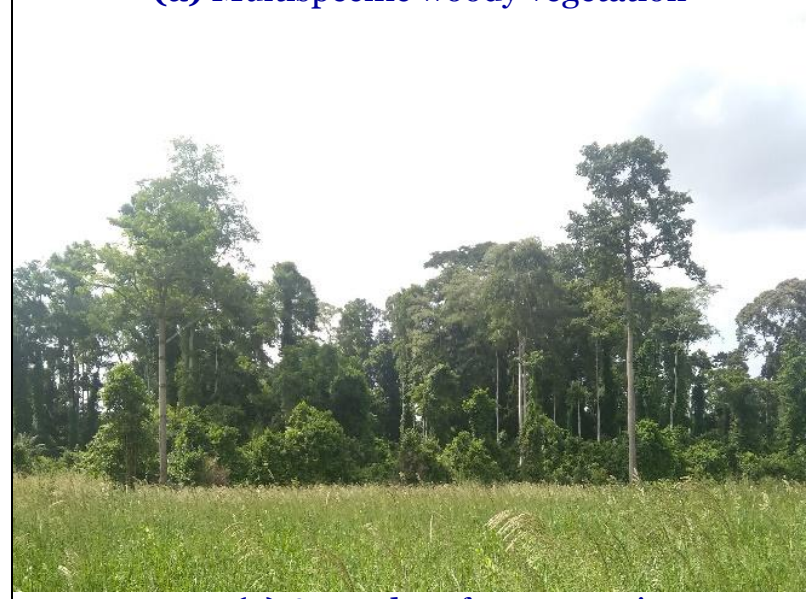

(c) Secondary forest mosaic

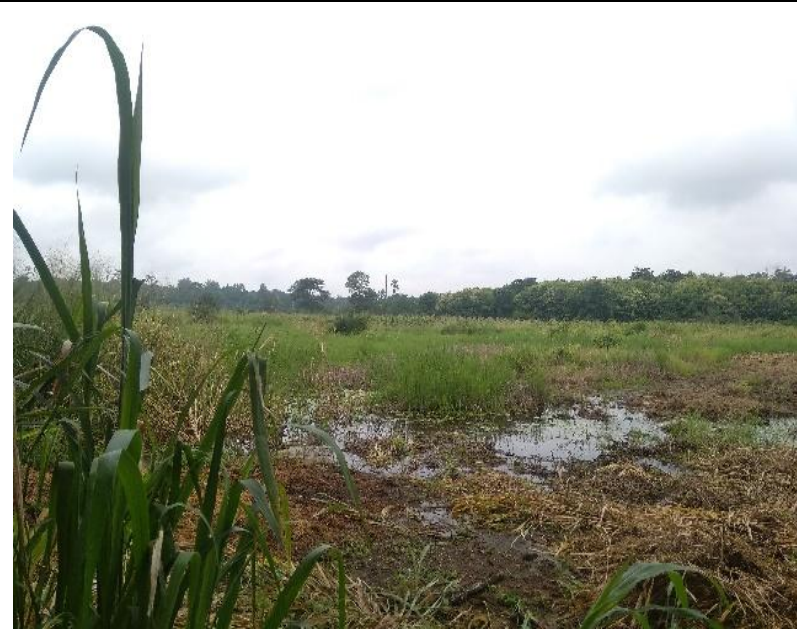

(b) Rice lowlands

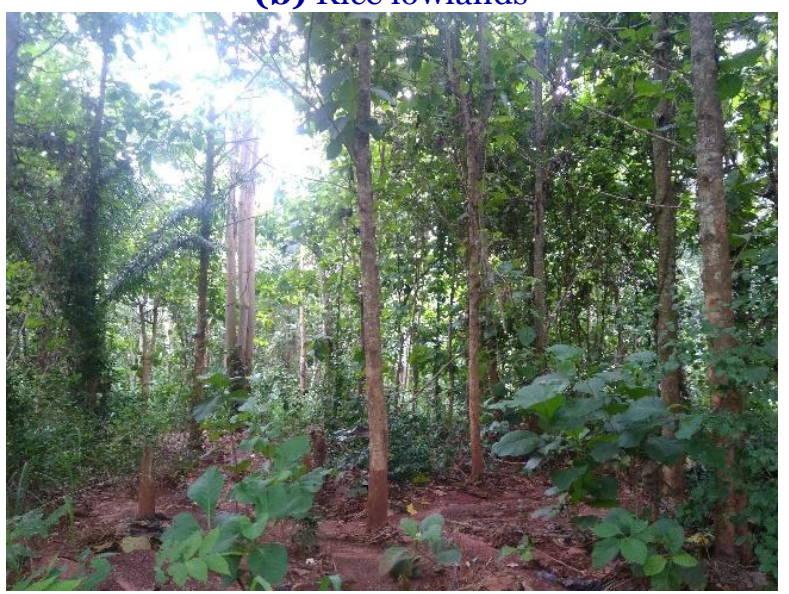

(d) Undergrowth of a monospecific teak plantation

Fig. 2: Partial views of some habitats of the BCF.

\section{Results}

\section{Avifauna richness}

In total, 150 bird species belonging to 50 families and 18 orders (Table 1) were inventoried in the different habitats of the BCF. This forest is home to $19.79 \%$ of Côte d'Ivoire's avifauna. Nonpasseriformes are the most important with 86 species (57.33\%) from 27 families. Within this group, the orders of Charadriiformes, Coraciiformes and Piciformes are the most diverse, with three families each. Next come the orders Bucerotiformes, Galliformes, Pelecaniformes and Strigiformes with two families each. With $62.96 \%$ of the families of the non-Passeriformes, these seven orders are the most important on this site. Passeriformes represent $37.04 \%$ of the settlement. The most diverse family in terms of number of species is the Accipitridae family with 14 species. 
The family Ploceidae is the second most diverse family with 13 species followed by the families Ardeidae and Cuculidae with eight species each. Then come the families of Lybiidae and
Pycnonotidae with seven species. These families alone account for $38 \%$ of the species richness. Fig. 3 shows some of the species inventoried in the BCF.

Table 1. List of bird species recorded in the BCF.

\begin{tabular}{|c|c|c|c|c|c|c|c|}
\hline NE & Scientific name & Common name & $\mathbf{S C}$ & Bio & HP & SB & $\mathbf{R R}$ \\
\hline & GALLIFORMES & & & & & & \\
\hline & NUMIDIDAE (1) & & & & & & \\
\hline 1 & $\begin{array}{l}\text { Numida meleagris (Linnaeus, 1758) } \\
\text { PHASIANIDAE (2) }\end{array}$ & Helmeted Guinea fowl & LC & & $\mathrm{f}$ & $\mathrm{R}$ & \\
\hline 2 & Peliperdix lathami (Hartlaub, 1854) & Latham's Forest Francolin & LC & GC & FF & $\mathrm{R}$ & \\
\hline 3 & $\begin{array}{l}\text { Pternistis bicalcaratus (Linnaeus, 1766) } \\
\text { ANSERIFORMES }\end{array}$ & Double-spurred Francolin & LC & & $\mathrm{f}$ & $\mathrm{R}$ & \\
\hline 4 & $\begin{array}{l}\text { ANATIDAE (1) } \\
\text { Dendrocygna viduata (Linnaeus, 1766) } \\
\text { COLUMBIFORMES } \\
\text { COLUMBIDAE (5) }\end{array}$ & White-faced Whistling Duck & LC & & Ea & $\mathrm{R}$ & \\
\hline 5 & Treron calvus (Temminck, 1811) & African Green Pigeon & LC & & $\mathrm{F}$ & $\mathrm{R}$ & \\
\hline 6 & Turtur brehmeri (Hartlaub, 1865) & Blue-headed Wood Dove & LC & GC & $\mathrm{F}$ & $\mathrm{R}$ & \\
\hline 7 & Turtur tympanistria (Temminck, 1809) & Tambourine Dove & LC & & $\mathrm{F}$ & $\mathrm{R}$ & \\
\hline 8 & Turtur afer (Linnaeus, 1766) & Blue-spotted Wood Dove & LC & & $\mathrm{f}$ & $\mathrm{R}$ & \\
\hline 9 & $\begin{array}{l}\text { Streptopelia semitorquata (Rüppell, 1837) } \\
\text { CAPRIMULGIFORMES } \\
\text { CAPRIMULGIDAE (3) }\end{array}$ & Red-eyed Dove & LC & & $\mathrm{f}$ & $\mathrm{R}$ & \\
\hline 10 & Caprimulgus longipennis (Shaw , 1796) & Standard-winged Nightjar & LC & & $\mathrm{f}$ & M & \\
\hline 11 & Caprimulgus europaeus (Linnaeus, 1758) & European Nightjar & LC & & $\mathrm{f}$ & $\mathrm{P}$ & \\
\hline 12 & $\begin{array}{l}\text { Caprimulgus tristigma (Rüppell, 1840) } \\
\text { CUCULIFORMES } \\
\text { CUCULIDAE (8) }\end{array}$ & Freckled Nightjar & LC & & $\mathrm{f}$ & $\mathrm{R}$ & \\
\hline 13 & Cuculus solitarius (Stephens , 1815) & Red-chested Cuckoo & LC & & $\mathrm{FF}$ & M & \\
\hline 14 & Cuculus clamosus (Latham, 1802) & Black Cuckoo & LC & & $\mathrm{FF}$ & M & \\
\hline 15 & Chrysococcyx cupreus (Shaw, 1792) & African Emerald Cuckoo & LC & & $\mathrm{F}$ & $\mathrm{R}$ & \\
\hline 16 & Chrysococcyx klaas (Stephens, 1815) & Klaas's Cuckoo & $\mathrm{LC}$ & & $\mathrm{f}$ & $\mathrm{R} / \mathrm{M}$ & \\
\hline 17 & Chrusococcyx caprius (Boddaert, 1783) & Didric Cuckoo & LC & & $\mathrm{f}$ & $\mathrm{R} / \mathrm{M}$ & \\
\hline 18 & Ceuthmochares aereus (Vieillot, 1817) & Yellow bill & LC & & $\mathrm{f}$ & $\mathrm{R}$ & \\
\hline 19 & Centropus grillii (Hartlaub, 1861) & Black Coucal & LC & & $\mathrm{f}$ & $\mathrm{M} / \mathrm{R}$ & \\
\hline 20 & $\begin{array}{l}\text { Centropus senegalensis (Linnaeus, 1766) } \\
\text { GRUIFORMES } \\
\text { RALLIDAE (4) }\end{array}$ & Senegal Coucal & LC & & $\mathrm{f}$ & $\mathrm{R}$ & \\
\hline 21 & Zapornia flavirostra (Swainson, 1837) & Black Crake & LC & & $\mathrm{Ea}$ & $\mathrm{R}$ & \\
\hline 22 & Porphyrio alleni (Thomson, 1842) & Allen's Gallinule & LC & & $\mathrm{Ea}$ & $\mathrm{R}$ & \\
\hline 23 & Porphyrio porphyrio (Linnaeus, 1758) & Purple Swamphen & LC & & $\mathrm{Ea}$ & $\mathrm{R} / \mathrm{M}$ & \\
\hline 24 & $\begin{array}{l}\text { Gallinula chloropus (Linnaeus, 1758) } \\
\text { MUSOPHAGIFORMES }\end{array}$ & Common Moorhen & LC & & $\mathrm{Ea}$ & $\mathrm{R}$ & \\
\hline & MUSOPHAGIDAE (3) & & & & & & \\
\hline 25 & Corythaeola cristata (Vieillot, 1816) & Great Blue Turaco & LC & & $\mathrm{FF}$ & $\mathrm{R}$ & \\
\hline 26 & Tauraco persa (Linnaeus, 1758) & Green Turaco & LC & GC & $\mathrm{FF}$ & $\mathrm{R}$ & \\
\hline 27 & $\begin{array}{l}\text { Crinifer piscator (Boddaert, 1783) } \\
\text { PELECANIFORMES } \\
\text { ARDEIDAE (8) }\end{array}$ & Western Grey Plantain-eater & LC & & $\mathrm{f}$ & $\mathrm{R}$ & \\
\hline 28 & Ixobrychus minutus (Linnaeus, 1766) & Little Bittern & LC & & $\mathrm{Ea}$ & $\mathrm{P}$ & \\
\hline 29 & Nycticorax nycticorax (Linnaeus, 1758) & Black-crowned Night Heron & LC & & $\mathrm{Ea}$ & $\mathrm{R}$ & \\
\hline 30 & Bubulcus ibis (Linnaeus, 1758) & Cattle Egret & LC & & $\mathrm{Ea}$ & $R / M$ & \\
\hline 31 & Butorides striata (Linnaeus, 1758) & Green-backed Heron & LC & & $\mathrm{Ea}$ & $\mathrm{R}$ & \\
\hline
\end{tabular}


Table 1 (Continued)...

\begin{tabular}{|c|c|c|c|c|c|c|c|}
\hline NE & Scientific name & Common name & SC & Bio & $\mathbf{H P}$ & SB & $\mathbf{R R}$ \\
\hline 32 & Egretta garzetta (Linnaeus, 1766) & Little Egret & LC & & $\mathrm{Ea}$ & $\mathrm{R}$ & \\
\hline 33 & Egretta intermedia (Wagler, 1829) & Intermediate Egret & LC & & $\mathrm{Ea}$ & $\mathrm{R}$ & \\
\hline 34 & Egretta alba (Linnaeus, 1758) & Great Egret & LC & & $\mathrm{Ea}$ & $\mathrm{R}$ & \\
\hline 35 & $\begin{array}{l}\text { Ardea cinerea (Linnaeus, 1758) } \\
\text { SCOPIDAE (1) }\end{array}$ & Grey Heron & $\mathrm{LC}$ & & $\mathrm{Ea}$ & $\mathrm{R} / \mathrm{P}$ & \\
\hline 36 & $\begin{array}{l}\text { Scopus umbretta (Gmelin, 1789) } \\
\text { SULIFORMES } \\
\text { PHALACROCORACIDAE (1) }\end{array}$ & Hamerkop & $\mathrm{LC}$ & & $\mathrm{Ea}$ & $\mathrm{R}$ & \\
\hline 37 & $\begin{array}{l}\text { Microcarbo africanus (Gmelin, 1789) } \\
\text { CHARADRIIFORMES } \\
\text { CHARADRIDAE (2) }\end{array}$ & Great Cormorant & $\mathrm{LC}$ & & Ea & $\mathrm{R}$ & \\
\hline 38 & Vanellus senegallus (Linnaeus, 1766) & African Wattled Lapwing & $\mathrm{LC}$ & & $\mathrm{Ea}$ & $\mathrm{R}$ & \\
\hline 39 & $\begin{array}{l}\text { Vanellus albiceps (Gould, 1834) } \\
\text { JACANIDAE (1) }\end{array}$ & White-headed Lapwing & LC & & Ea & M & \\
\hline 40 & $\begin{array}{l}\text { Actophilornis africanus (Gmelin, 1789) } \\
\text { SCOLOPACIDAE (2) }\end{array}$ & African Jacana & $\mathrm{LC}$ & & $\mathrm{Ea}$ & $\mathrm{R}$ & \\
\hline 41 & Tringa glareola (Linnaeus, 1758) & Wood Sandpiper & LC & & $\mathrm{Ea}$ & $\mathrm{P}$ & \\
\hline 42 & $\begin{array}{l}\text { Actitis hypoleucos (Linnaeus, 1758) } \\
\text { STRIGIFORMES } \\
\text { TYTONIDAE (1) }\end{array}$ & Common Sandpiper & LC & & $\mathrm{Ea}$ & $\mathrm{P}$ & \\
\hline 43 & $\begin{array}{l}\text { Tyto alba (Scopoli, 1769) } \\
\text { STRIGIDAE (3) }\end{array}$ & Barn Owl & LC & & $\mathrm{f}$ & $\mathrm{R}$ & \\
\hline 44 & Ptilopsis leucotis (Temminck, 1820) & White-faced Owl & $\mathrm{LC}$ & & $\mathrm{f}$ & $\mathrm{R}$ & \\
\hline 45 & Jubula lettii (Büttikofer, 1889) & Maned Owl & DD & GC & $\mathrm{FF}$ & $\mathrm{R}$ & \\
\hline 46 & $\begin{array}{l}\text { Strix woodfordii (Smith, 1834) } \\
\text { ACCIPITRIFORMES } \\
\text { ACCIPITRIDAE (14) }\end{array}$ & African Wood Owl & LC & & $\mathrm{F}$ & $\mathrm{R}$ & \\
\hline 47 & Pernis apivorus (Linnaeus, 1758) & European Honey Buzzard & $\mathrm{LC}$ & & $\mathrm{f}$ & $\mathrm{P}$ & \\
\hline 48 & $\begin{array}{l}\text { Macheiramphus alcinus (Westermann, } \\
\text { 1851) }\end{array}$ & Bat Hawk & $\mathrm{LC}$ & & $\mathrm{F}$ & $\mathrm{R}$ & \\
\hline 49 & Elanus caeruleus (Desfontaines, 1789) & Black-shouldered Kite & $\mathrm{LC}$ & & $\mathrm{f}$ & $\mathrm{R}$ & \\
\hline 50 & Milvus migrans (Boddaert, 1783) & Black Kite & $\mathrm{LC}$ & & $\mathrm{f}$ & M & \\
\hline 51 & Gypohierax angolensis (Gmelin, 1788) & Palm-nut Vulture & LC & & $\mathrm{F}$ & $\mathrm{R}$ & \\
\hline 52 & Polyboroides typus (Smith, 1829) & African Harrier Hawk & LC & & $\mathrm{F}$ & $\mathrm{R}$ & \\
\hline 53 & Circus macrourus (Gmelin, 1770) & Pallid Harrier & NT & & $\mathrm{f}$ & $\mathrm{P}$ & \\
\hline 54 & Micronisus gabar (Daudin, 180o) & Gabar Goshawk & LC & & $\mathrm{f}$ & $\mathrm{R}$ & \\
\hline 55 & Accipiter tachiro (Daudin, 180o) & African Goshawk & $\mathrm{LC}$ & & $\mathrm{FF}$ & $\mathrm{R}$ & \\
\hline 56 & Accipiter badius (Gmelin, 1788) & Shikra & LC & & $\mathrm{f}$ & $\mathrm{R}$ & \\
\hline 57 & $\begin{array}{l}\text { Kaupifalco monogrammicus (Temminck, } \\
1824 \text { ) }\end{array}$ & Lizard Buzzard & LC & & $\mathrm{f}$ & $\mathrm{R}$ & \\
\hline 58 & Buteo auguralis (Salvadori, 1865) & Red-necked Buzzard & LC & & $\mathrm{f}$ & $\mathrm{R} / \mathrm{M}$ & \\
\hline 59 & Aquila rapax (Temminck, 1828) & Tawny Eagle & LC & & $\mathrm{f}$ & $\mathrm{R}$ & \\
\hline 60 & Hieraaetus ayresii (Gurney, 1862) & Ayres's Hawk Eagle & $\mathrm{LC}$ & & FF & $\mathrm{R}$ & \\
\hline & $\begin{array}{l}\text { BUCEROTIFORMES } \\
\text { BUCEROTIDAE (4) }\end{array}$ & & & & & & \\
\hline 61 & Lophoceros semifasciatus (Hartlaub, 1855) & African Pied Hornbill & LC & GC & $\mathrm{F}$ & $\mathrm{R}$ & \\
\hline 62 & Lophocerus nasutus (Linnaeus, 1766) & African Grey Hornbill & LC & & $\mathrm{f}$ & $\mathrm{R}$ & \\
\hline 63 & $\begin{array}{l}\text { Bycanistes fistulator (Cassin, 1852) } \\
\text { UPUPIDAE (1) }\end{array}$ & Piping Hornbill & LC & GC & $\mathrm{f}$ & $\mathrm{R}$ & \\
\hline 64 & $\begin{array}{l}\text { Upupa epops (Linnaeus, 1758) } \\
\text { CORACIIFORMES } \\
\text { MEROPIDAE (2) }\end{array}$ & Hoopoe & $\mathrm{LC}$ & & $\mathrm{f}$ & $\mathrm{M} / \mathrm{P}$ & \\
\hline 65 & Merops albicollis (Vieillot, 1817) & White-throated Bee-eater & LC & & $\mathrm{f}$ & M & \\
\hline 66 & Merops apiaster (Linnaeus, 1758) & European Bee-eater & LC & & $\mathrm{f}$ & $\mathrm{P}$ & \\
\hline
\end{tabular}


Table 1 (Continued)...

\begin{tabular}{|c|c|c|c|c|c|c|c|}
\hline NE & Scientific name & Common name & SC & Bio & HP & SB & $\mathbf{R R}$ \\
\hline & CORACIIDAE (3) & & & & & & \\
\hline 67 & Coracias cyanogaster (Cuvier, 1817) & Blue-bellied Roller & $\mathrm{LC}$ & SG & $\mathrm{f}$ & $\mathrm{R}$ & \\
\hline 68 & Eurystomus gularis (Vieillot, 1819) & Blue-throated Roller & LC & GC & $\mathrm{F}$ & $\mathrm{R}$ & \\
\hline 69 & $\begin{array}{l}\text { Eurystomus glaucurus (Müller, 1776) } \\
\text { ALCEDINIDAE (4) }\end{array}$ & Broad-billed Roller & $\mathrm{LC}$ & & $\mathrm{f}$ & $\mathrm{R} / \mathrm{M}$ & \\
\hline 70 & Halcyon senegalensis (Linnaeus, 1766) & Woodland Kingfisher & $\mathrm{LC}$ & & $\mathrm{f}$ & $\mathrm{R}$ & \\
\hline 71 & Ispidina pictus (Boddaert, 1783) & African Pygmy Kingfisher & $\mathrm{LC}$ & & $\mathrm{f}$ & $\mathrm{R}$ & \\
\hline 72 & Corythornis leucogaster (Fraser, 1843) & White-bellied Kingfisher & $\mathrm{LC}$ & GC & $\mathrm{f}$ & $\mathrm{R}$ & \\
\hline 73 & $\begin{array}{l}\text { Corythornis cristatus (Pallas, 1764) } \\
\text { PICIFORMES } \\
\text { LYBIIDAE ( } 7 \text { ) }\end{array}$ & Malachite Kingfisher & $\mathrm{LC}$ & & Ea & $\mathrm{R}$ & \\
\hline 74 & Gymnobucco calvus (Lafresnaye, 1841) & Naked-faced Barbet & $\mathrm{LC}$ & GC & $\mathrm{F}$ & $\mathrm{R}$ & \\
\hline 75 & Pogoniulus scolopaceus (Bonaparte, 1850) & Speckled Tinkerbird & $\mathrm{LC}$ & GC & $\mathrm{F}$ & $\mathrm{R}$ & \\
\hline 76 & Pogoniulus subsulphureus (Fraser, 1843) & Yellow-throated Tinkerbird & $\mathrm{LC}$ & GC & FF & $\mathrm{R}$ & \\
\hline 77 & Pogoniulus bilineatus (Sundevall, 1850) & Yellow-rumped Tinkerbird & $\mathrm{LC}$ & & $\mathrm{F}$ & $\mathrm{R}$ & \\
\hline 78 & Tricholaema hirsuta (Swainson, 1821) & Hairy-breasted Barbet & $\mathrm{LC}$ & GC & $\mathrm{F}$ & $\mathrm{R}$ & \\
\hline 79 & Lybius vieilloti (Leach, 1815) & Vieillot's Barbet & $\mathrm{LC}$ & & $\mathrm{f}$ & $\mathrm{R}$ & \\
\hline 80 & $\begin{array}{l}\text { Pogonornis bidentatus (Shaw, 1798) } \\
\text { INDICATORIDAE (1) }\end{array}$ & Double-toothed Barbet & $\mathrm{LC}$ & & $\mathrm{f}$ & $\mathrm{R}$ & \\
\hline 81 & $\begin{array}{l}\text { Indicator indicator (Sparrman, 1777) } \\
\text { PICIDAE (1) }\end{array}$ & Greater Honeyguide & $\mathrm{LC}$ & & f & $\mathrm{R}$ & \\
\hline 82 & $\begin{array}{l}\text { Dendropicos pyrrhogaster (Malherbe, } \\
\text { 1845) } \\
\text { FALCONIFORMES } \\
\text { FALCONIDAE (2) }\end{array}$ & Fire-bellied Woodpecker & $\mathrm{LC}$ & GC & $\mathrm{F}$ & $\mathrm{R}$ & \\
\hline 83 & Falco ardosiaceus (Vieillot, 1823) & Grey Kestrel & $\mathrm{LC}$ & & $\mathrm{f}$ & $\mathrm{R}$ & \\
\hline 84 & $\begin{array}{l}\text { Falco biarmicus (Temminck, 1825) } \\
\text { PSITTACIFORMES } \\
\text { PSITTACIDAE (2) }\end{array}$ & Lanner Falcon & $\mathrm{LC}$ & & $\mathrm{f}$ & $\mathrm{R}$ & \\
\hline 85 & Psittacus timneh (Fraser, 1844) & Grey Parrot & EN & & $\mathrm{FF}$ & $\mathrm{R}$ & \\
\hline 86 & $\begin{array}{l}\text { Poicephalus gulielmi (Jardine, 1849) } \\
\text { PASSERIFORMES } \\
\text { ORIOLIDAE (1) }\end{array}$ & Red-fronted Parrot & $\mathrm{LC}$ & & $\mathrm{FF}$ & $\mathrm{R}$ & \\
\hline 87 & $\begin{array}{l}\text { Oriolus brachyrhynchus (Swainson, 1837) } \\
\text { CAMPEPHAGIDAE (2) }\end{array}$ & Western Black-headed Oriole & $\mathrm{LC}$ & GC & $\mathrm{F}$ & $\mathrm{R}$ & \\
\hline 88 & Ceblepyris pectoralis (Temminck, 1824) & White-breasted Cuckoo-shrike & $\mathrm{LC}$ & & $\mathrm{f}$ & $\mathrm{R}$ & \\
\hline 89 & $\begin{array}{l}\text { Cyanograucalus azureus (Cassin, 1852) } \\
\text { VANGIDAE (1) }\end{array}$ & Blue Cuckoo-shrike & $\mathrm{LC}$ & GC & $\mathrm{FF}$ & $\mathrm{R}$ & \\
\hline 90 & $\begin{array}{l}\text { Dyaphorophyia castanea (Fraser, 1843) } \\
\text { PLATYSTEIRIDAE (1) }\end{array}$ & Chestnut Wattle-eye & $\mathrm{LC}$ & GC & $\mathrm{FF}$ & $\mathrm{R}$ & \\
\hline 91 & $\begin{array}{l}\text { Platysteira cyanea (Müller, 1776) } \\
\text { MALACONOTIDAE (2) }\end{array}$ & Common Wattle-eye & $\mathrm{LC}$ & & $\mathrm{f}$ & $\mathrm{R}$ & \\
\hline 92 & Tchagra australis (Smith, 1836) & Brown-crowned Tchagra & $\mathrm{LC}$ & & $\mathrm{F}$ & $\mathrm{R}$ & \\
\hline 93 & $\begin{array}{l}\text { Tchagra senegalus (Linnaeus, 1766) } \\
\text { DICRURIDAE (2) }\end{array}$ & Black-crowned Tchagra & $\mathrm{LC}$ & & $\mathrm{f}$ & $\mathrm{R}$ & \\
\hline 94 & Dicrurus adsimilis(Bechstein, 1794) & Fork-tailed Drongo & $\mathrm{LC}$ & & $\mathrm{F}$ & $\mathrm{R}$ & \\
\hline 95 & $\begin{array}{l}\text { Dicrurus modestus (Hartlaub, 1849) } \\
\text { MONARCHIDAE (1) }\end{array}$ & Velvet-mantled Drongo & $\mathrm{LC}$ & & $\mathrm{F}$ & $\mathrm{R}$ & \\
\hline 96 & Terpsiphone rufiventer (Müller, 1776) & $\begin{array}{l}\text { Red-bellied Paradise } \\
\text { Flycatcher }\end{array}$ & $\mathrm{LC}$ & GC & $\mathrm{F}$ & $\mathrm{R}$ & \\
\hline 97 & $\begin{array}{l}\text { LANIIDAE (1) } \\
\text { Lanius collaris (Linnaeus, 1766) } \\
\text { CORVIDAE (1) }\end{array}$ & Common Fiscal & $\mathrm{LC}$ & & $\mathrm{f}$ & $\mathrm{R}$ & \\
\hline 98 & Corvus albus (Müller, 1776) & Pied Crow & $\mathrm{LC}$ & & $\mathrm{f}$ & $\mathrm{R}$ & \\
\hline
\end{tabular}


Table 1 (Continued)...

\begin{tabular}{|c|c|c|c|c|c|c|c|}
\hline NE & Scientific name & Common name & SC & Bio & HP & SB & $\mathbf{R R}$ \\
\hline 99 & $\begin{array}{l}\text { MACROSPHENIDAE (1) } \\
\text { Sylvietta virens (Cassin, 1859) } \\
\text { CISTICOLIDAE (9) }\end{array}$ & Green Crombec & $\mathrm{LC}$ & GC & $\mathrm{F}$ & $\mathrm{R}$ & \\
\hline 100 & Camaroptera brachyura (Vieillot, 1820) & Grey-backed Camaroptera & $\mathrm{LC}$ & & f & $\mathrm{R}$ & \\
\hline 101 & Camaroptera chloronota (Reichenow, 1895) & Olive-green Camaroptera & $\mathrm{LC}$ & GC & $\mathrm{FF}$ & $\mathrm{R}$ & \\
\hline 102 & Cisticola erythrops (Hartlaub, 1857) & Red-faced Cisticola & $\mathrm{LC}$ & & $\mathrm{f}$ & $\mathrm{R}$ & \\
\hline 103 & Cisticola cantans (Heuglin, 1869) & Singing Cisticola & $\mathrm{LC}$ & & $\mathrm{f}$ & $\mathrm{R}$ & \\
\hline 104 & Cisticola lateralis (Fraser, 1843) & Whistling Cisticola & $\mathrm{LC}$ & & $\mathrm{f}$ & $\mathrm{R}$ & \\
\hline 105 & Cisticola galactotes (Temminck, 1821) & Winding Cisticola & $\mathrm{LC}$ & & $\mathrm{f}$ & $\mathrm{R}$ & \\
\hline 106 & Cisticola brachypterus (Sharpe, 1870) & Short-winged Cisticola & $\mathrm{LC}$ & & $\mathrm{f}$ & $\mathrm{R}$ & \\
\hline 107 & $\begin{array}{l}\text { Prinia subflava (Gmelin, 1789) } \\
\text { HIRUNDINIDAE (2) }\end{array}$ & Tawny-flanked Prinia & $\mathrm{LC}$ & & $\mathrm{f}$ & $\mathrm{R}$ & \\
\hline 108 & $\begin{array}{l}\text { Cecropis abyssinica (Guérin-Méneville, } \\
\text { 1843) }\end{array}$ & Lesser Striped Swallow & $\mathrm{LC}$ & & $\mathrm{f}$ & $\mathrm{R}$ & \\
\hline 109 & $\begin{array}{l}\text { Hirundo rustica (Linnaeus, 1758) } \\
\text { PYCNONOTIDAE (7) }\end{array}$ & Barn Swallow & $\mathrm{LC}$ & & f & $\mathrm{P}$ & \\
\hline 110 & Stelgidillas gracilirostris (Strickland, 1844) & Slender-billed Greenbul & LC & & $\mathrm{FF}$ & $\mathrm{R}$ & \\
\hline 111 & Bleda canicapillus (Hartlaub, 1854) & Grey-headed Bristlebill & LC & GC & FF & $\mathrm{R}$ & \\
\hline 112 & Thescelocichla leucopleura (Cassin, 1856) & Swamp Palm Bulbul & $\mathrm{LC}$ & GC & $\mathrm{F}$ & $\mathrm{R}$ & \\
\hline 113 & Chlorocichla simplex (Hartlaub, 1855) & Simple Leaflove & $\mathrm{LC}$ & GC & $\mathrm{F}$ & $\mathrm{R}$ & \\
\hline 114 & Eurillas latirostris (Strickland, 1844) & Yellow-whiskered Greenbul & $\mathrm{LC}$ & & $\mathrm{F}$ & $\mathrm{R}$ & \\
\hline 115 & Eurillas virens (Cassin, 1858) & Little Greenbul & $\mathrm{LC}$ & & $\mathrm{F}$ & $\mathrm{R}$ & \\
\hline 116 & $\begin{array}{l}\text { Pycnonotus barbatus (Desfontaine, 1789) } \\
\text { SCOTOCERCIDAE (1) }\end{array}$ & Common Bulbul & $\mathrm{LC}$ & & $\mathrm{f}$ & $\mathrm{R}$ & \\
\hline 117 & $\begin{array}{l}\text { Hylia prasina (Cassin, } 1855) \\
\text { STURNIDAE (2) }\end{array}$ & Green Hylia & $\mathrm{LC}$ & GC & f & $\mathrm{R}$ & \\
\hline 118 & Onychognathus fulgidus (Hartlaub, 1849) & $\begin{array}{l}\text { Forest Chestnut-winged } \\
\text { Starling }\end{array}$ & $\mathrm{LC}$ & GC & $\mathrm{F}$ & $\mathrm{R}$ & \\
\hline 119 & $\begin{array}{l}\text { Lamprotornis splendidus (Vieillot, 1822) } \\
\text { MUSCICAPIDAE (2) }\end{array}$ & Splendid Glossy Starling & $\mathrm{LC}$ & & $\mathrm{F}$ & $\mathrm{R}$ & \\
\hline 120 & $\begin{array}{l}\text { Melaenornis annamarulae (Forbes- } \\
\text { Watson, } 1970\end{array}$ & Nimba Flycatcher & VU & GC & $\mathrm{FF}$ & $\mathrm{R}$ & $\mathrm{RR}$ \\
\hline 121 & $\begin{array}{l}\text { Saxicola rubetra (Linnaeus, 1758) } \\
\text { NECTARINIIDAE (6) }\end{array}$ & African [Common] Stonechat & $\mathrm{LC}$ & & f & $\mathrm{P}$ & \\
\hline 122 & Hedydipna collaris (Vieillot, 1819) & Collared Sunbird & $\mathrm{LC}$ & & $\mathrm{f}$ & $\mathrm{R}$ & \\
\hline 123 & Cyanomitra olivacea (Smith, 1840) & Olive Sunbird & LC & & FF & $\mathrm{R}$ & \\
\hline 124 & Chalcomitra adelberti (Gervais, 1833) & Buff-throated Sunbird & LC & GC & $\mathrm{F}$ & $\mathrm{R}$ & \\
\hline 125 & Cinnyris chloropygius (Jardine, 1842) & Olive-bellied Sunbird & $\mathrm{LC}$ & & $\mathrm{f}$ & $\mathrm{R}$ & \\
\hline 126 & Cinnyris coccinigastrus (Latham, 1801) & Splendid Sunbird & $\mathrm{LC}$ & SG & $\mathrm{f}$ & $\mathrm{R}$ & \\
\hline 127 & $\begin{array}{l}\text { Cinnyris cupreus (Shaw, 1811) } \\
\text { PLOCEIDAE (13) }\end{array}$ & Copper Sunbird & $\mathrm{LC}$ & & $\mathrm{f}$ & $\mathrm{R}$ & \\
\hline 128 & Amblyospiza albifrons (Vigors, 1831) & Grosbeak Weaver & $\mathrm{LC}$ & & $\mathrm{f}$ & $\mathrm{R}$ & \\
\hline 129 & Quelea erythrops (Hartlaub, 1848) & Red-headed Quelea & $\mathrm{LC}$ & & $\mathrm{f}$ & $\mathrm{R} / \mathrm{M}$ & \\
\hline 130 & Quelea quelea (Linnaeus, 1758) & Red-billed Quelea & $\mathrm{LC}$ & & $\mathrm{f}$ & $\mathrm{O}$ & \\
\hline 131 & Euplectes hordeaceus (Linnaeus, 1758) & Black-winged Red Bishop & LC & & f & $\mathrm{R}$ & \\
\hline 132 & Euplectes macroura (Gmelin, 1789) & Yellow-mantled Widowbird & LC & & $\mathrm{f}$ & $\mathrm{R}$ & \\
\hline 133 & Ploceus nigricollis (Vieillot, 1805) & Black-necked Weaver & $\mathrm{LC}$ & & $\mathrm{f}$ & $\mathrm{R}$ & \\
\hline 134 & Ploceus cucullatus (Müller, 1776) & Village Weaver & $\mathrm{LC}$ & & $\mathrm{f}$ & $\mathrm{R}$ & \\
\hline 135 & Ploceus tricolor (Hartlaub, 1854) & Yellow-mantled Weaver & $\mathrm{LC}$ & GC & FF & $\mathrm{R}$ & \\
\hline 136 & Ploceus superciliosus (Shelley, 1873) & Compact Weaver & $\mathrm{LC}$ & & $\mathrm{f}$ & $\mathrm{R}$ & \\
\hline 137 & $\begin{array}{l}\text { Ploceus albinucha (Barboza du Bocage, } \\
\text { 1876) }\end{array}$ & Maxwell's Black Weaver & $\mathrm{LC}$ & & $\mathrm{FF}$ & $\mathrm{R}$ & \\
\hline 138 & Ploceus nigerrimus (Vieillot, 1819) & Vieillot's Black Weaver & LC & GC & $\mathrm{f}$ & $\mathrm{R}$ & \\
\hline 139 & Malimbus nitens (Gray, 1831) & Blue-billed Malimbe & $\mathrm{LC}$ & GC & $\mathrm{F}$ & $\mathrm{R}$ & \\
\hline 140 & Malimbus rubricollis (Swainson, 1838) & Red-headed Malimbe & $\mathrm{LC}$ & GC & $\mathrm{f}$ & $\mathrm{R}$ & \\
\hline
\end{tabular}


Table 1 (Continued)...

\begin{tabular}{|c|c|c|c|c|c|c|c|}
\hline NE & Scientific name & Common name & SC & Bio & HP & SB & $\mathbf{R R}$ \\
\hline & ESTRILDIDAE (5) & & & & & & \\
\hline 141 & Lagonosticta rubricata (Lichtenstein, 1823) & Blue-billed Firefinch & $\mathrm{LC}$ & & $\mathrm{F}$ & $\mathrm{R}$ & \\
\hline 142 & Spermophaga haematina (Vieillot, 1805) & Western Bluebill & LC & GC & $\mathrm{f}$ & $\mathrm{R}$ & \\
\hline 143 & Estrilda melpoda (Vieillot, 1817) & Orange-cheeked Waxbill & LC & & $\mathrm{f}$ & $\mathrm{R}$ & \\
\hline 144 & Spermestes cucullatus (Swainson, 1837) & Bronze Mannikin & LC & & $\mathrm{f}$ & $\mathrm{R}$ & \\
\hline 145 & $\begin{array}{l}\text { Spermestes bicolor (Fraser, 1843) } \\
\text { VIDUIDAE (3) }\end{array}$ & Black-and-white Mannikin & $\mathrm{LC}$ & & $\mathrm{f}$ & $\mathrm{R}$ & \\
\hline 146 & $\begin{array}{l}\text { Vidua macroura (Pallas, 1764) } \\
\text { PASSERIDAE (1) }\end{array}$ & Pin-tailed Whydah & LC & & $\mathrm{f}$ & $\mathrm{R}$ & \\
\hline 147 & Passer griseus (Vieillot, 1817) & $\begin{array}{l}\text { Northern Grey-headed } \\
\text { Sparrow }\end{array}$ & LC & & $\mathrm{f}$ & $\mathrm{R}$ & \\
\hline & MOTACILLIDAE (3) & & & & & & \\
\hline 148 & Anthus trivialis (Linnaeus, 1758) & Tree Pipit & LC & & $\mathrm{f}$ & $\mathrm{P}$ & \\
\hline 149 & $\begin{array}{l}\text { Anthus leucophrys (Vieillot, 1818) } \\
\text { FRINGILLIDAE (1) }\end{array}$ & Plain-backed Pipit & LC & & $\mathrm{f}$ & $\mathrm{R}$ & \\
\hline 150 & Crithagra mozambicus (Müller, 1776) & Yellow-fronted Canary & LC & & $\mathrm{f}$ & $\mathrm{R}$ & \\
\hline
\end{tabular}

Bio: Biome; DD: Data Deficient ; Ea: Wetland; EN: Endangered; F: General forest specialist; f: Open environment; FF: Forest specialist; BCF: Bouaflé Classified Forest ; Fr: Relative frequency ; GC : Guinean-Congolese forest biome species; HP: Preferred habitat; LC: Least concern; M: Intra-African migratory; NE: Species serial number; NT: Near threatened; P: Palearctic migratory; R: Resident; Re: Regular ; RR: Restricted distribution; SB: Biogeographic status; SC : Conservation status; SG: Sudano-Guinean savannah biome species; VU: Vulnerable.

\section{Characterisation of the population}

In terms of preferred habitats, the $\mathrm{CBF}$ has 56 species (37.33\%) in open habitats (F), 43 species (28.67\%) general forest specialists (F), 28 species (18.67\%) forest specialists (FF) and 23 species (15.33\%) in wetlands (E). The birds of the BCF are of different bio-geographical origins, but are predominantly Resident, with 123 species (82\%). Ten species (6.67\%) are Intra-African migratory (M) and six (6.67\%) species are Palearctic migratory $(\mathrm{P})$. Ten species $(6.67 \%)$ have a mixed status $(7 \mathrm{R} / \mathrm{M} ; 1 \mathrm{R} / \mathrm{P} ; 1 \mathrm{M} / \mathrm{R})$ and one species (0.66\%) is casual (O). Only one species (0.66\%) is casual in BCF.

\section{Species of conservation interest}

According to the IUCN status, three species on the Red List of Threatened Species have been observed there. These are a species close to threatened or Near threatened (NT) the Pallid Harrier Circus marourus (Gmelin, 1770), a Vulnerable species (VU) the Nimba Flycatcher Melaenornis annamarulae (Forbes-Watson, 1970) and an Endangered species (EN) the Grey Parrot Psittacus timneh (Fraser, 1844). Scientific Data deficient (DD) for the Maned Owl Jubula lettii. The other species are of Least Concern (LC). The BCF is home to two bird species belonging to the Sudano-Guinean savannah biomes, 31 species from the Guinean-Congolese forest biomes and one Restricted - range species.

\section{Discussion}

The $\mathrm{BCF}$ is rich and diverse with 150 species. This number of species could be explained by the combination of the sampling techniques used during this study (observation, listening to cries and songs, capture and recapture with mist nets and the vocalization replay technique). These observations are also made by Yaokokoré-Beibro (2010) during studies of avifaunal diversity in the Besso classified forest in south-eastern Côte d'Ivoire. For a shorter period, the N'ganda N'ganda Classified Forest (Kouadio et al., 2014), the Téné Classified Forest (Yaokokoré-Béibro et al., 2015) and the Haut Bandama Flora and Fauna Reserve (Ahon et al., 2020) contain 132, 93 and 178 bird species respectively. The latter share 82, 64 and 86 species with $\mathrm{BCF}$, respectively. This homogeneity between the Classified Forests in terms of species richness reflects the state of degradation of most protected areas in Côte d'Ivoire. The predominance of open habitat species $(37.33 \%)$ highlights the state of degradation of the BCF. Indeed, this forest relic is fragmented throughout the year mainly by anthropogenic activities such as logging and 
intensive and extensive agriculture, thus simultaneously reducing forest cover and nesting sites for forest specialist bird species. The presence of the Pallid Harrier Circus marourus (Gmelin, 1770), the Nimba Flycatcher Melaenornis annamarulae (Forbes-Watson, 1970) and the Grey
Parrot Psittacus timneh (Fraser, 1844), whose protection is of global interest (IUCN, 2020), 31 species endemic to the Guinean-Congolese forest biomes, and two species that belong to the SudanoGuinean savannah show that BCF in full degradation deserves special attention.

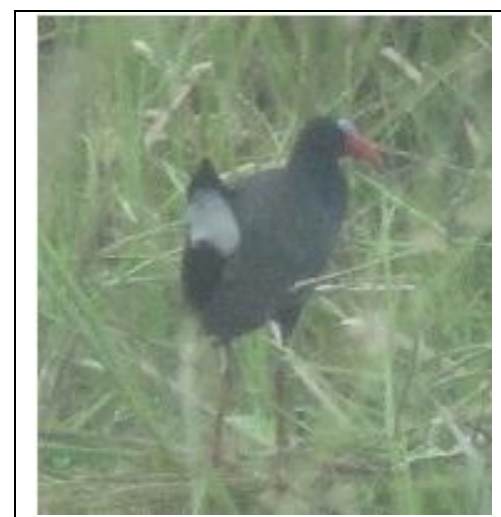

(a) Allen's Gallinule Porphyrio alleni

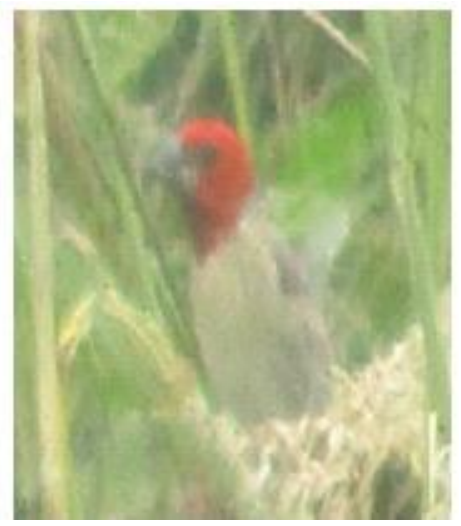

(d) Red-headed Quelea Quelea erythrops

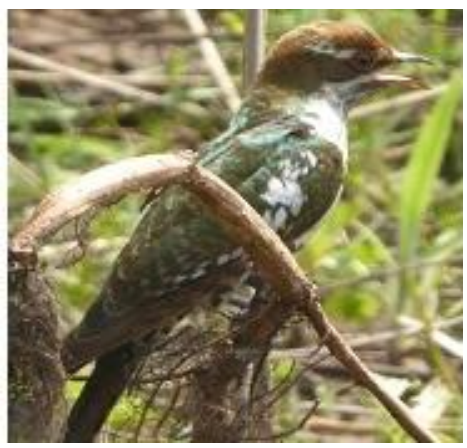

(g) Didric Cuckoo Chrysococcyx caprius

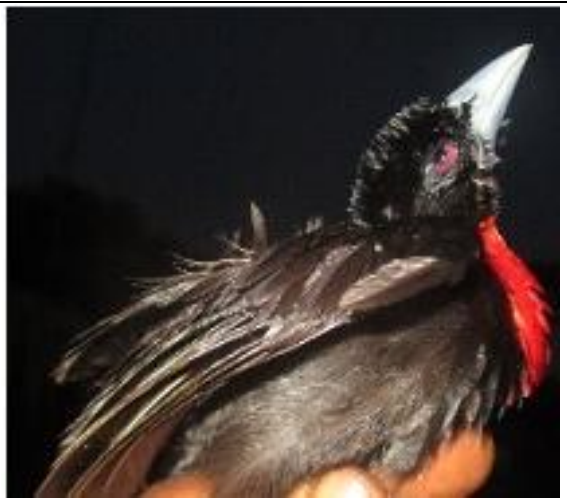

(b) Blue-billed Malimbe Malimbus nitens

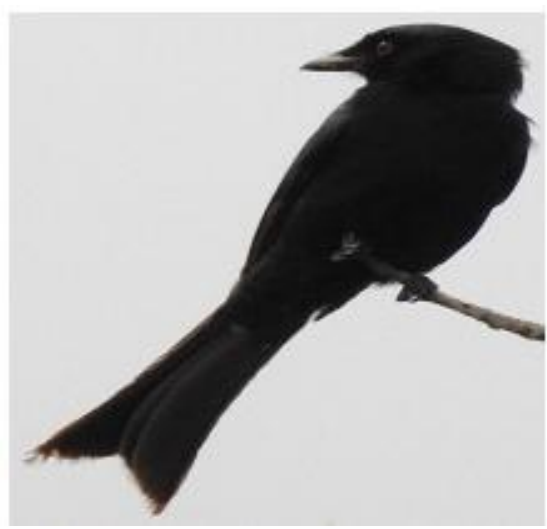

(e) Velvet-mantled Drongo Dicrurus modestus

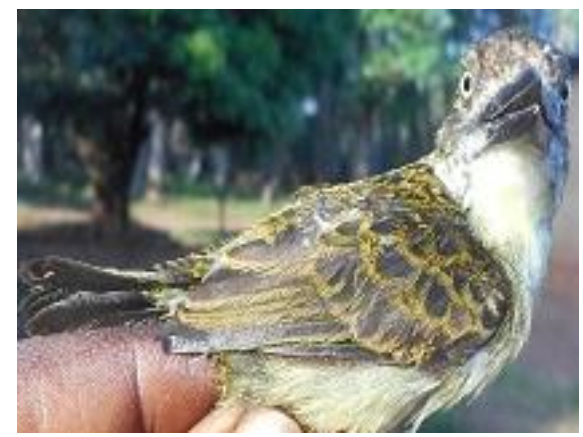

(h) Speckled Tinkerbird Pogoniulus scolopaceus

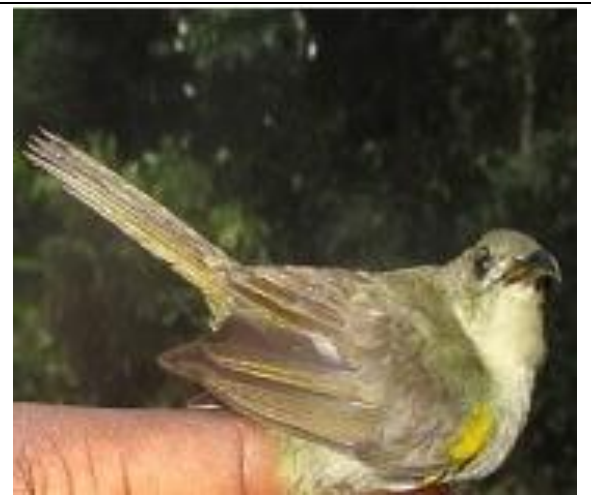

(c) Olive sunbird Cyanomitra olivacea

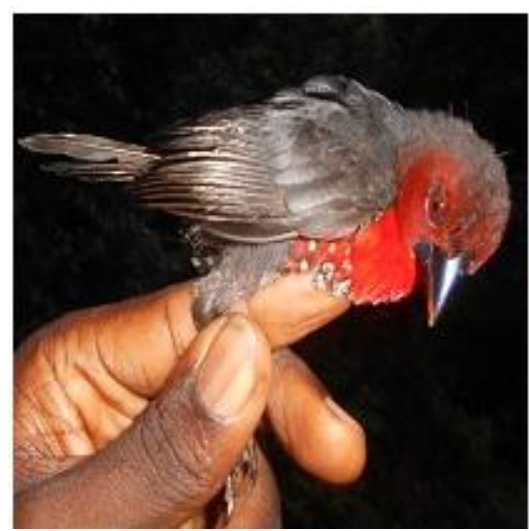

(f) Western Bluebill Spermophaga haematina

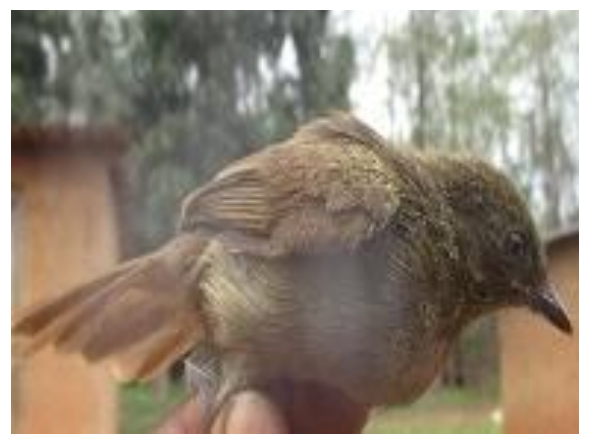

(i) Little green bul Eurillas virens

Fig. 3: Some bird species encountered in the BCF. 
Strictly forest species whose range covers the study area according to Borrow and Demey (2001) could not be observed. For example, these are the Western Bronze-naped Pigeon Columba iriditorques, Black-throated Coucal Centropus leucogaster, Yellow-billed Turaco Tauraco macrorhynchus, Congo Serpent Eagle Dryotriorchis spectabilis, Red-thighed Sparrowhawk Accipiter erythropus, of Cassin's Hawk Eagle Spizaetus africanus, Black Dwarf Hornbill Horizoceros hartlaubi, Red-billed Dwarf Hornbill Lophoceros camurus, Black-and-wcasqued Hornbill Bycanistes subcylindricus and Yellow-spotted Barbet Buccanodon duchaillui. Their absence could be attributed mainly to manmade activities. Generally speaking, deforestation therefore leads to the disappearance of more specialised forest species, leaving the place to generalist species that are better adapted to the unfavourable conditions of the environment, but up to a certain tolerance limit where these generalist species would start to disappear progressively. Thus, forest unit managers should integrate the ecological monitoring of wild fauna into their management plans for sustainable ecosystem management.

\section{Conclusion}

A study of the avifaunal population of the classified forest of Bouafle has revealed that it is rich and diversified with 150 species from 48 families and 18 orders. This diversity is related to the ecological structure of the different habitats. This study has made it possible to improve knowledge of the general state of conservation of this forest relic. However, the greater representation of species from open environments and the presence of threatened species leaves us somewhat perplexed. In fact, together with the local populations, the Société de Développement des Forêts (SODEFOR), which is responsible for managing this forest, must undertake large-scale actions to preserve this heritage. It is therefore recommend that additional studies be carried out on the effects of reforestation on birds and other wildlife groups (mammals, reptiles, amphibians, insects, and fish) for a better understanding of the biodiversity of this forest. These data will be made available to forest managers so that the wildlife component can be better taken into account in the management plan. In this context, raising the awareness and training of local populations will be an asset for the sustainable management of the forest's natural resources.

\section{Conflict of interest statement}

Authors declare that they have no conflict of interest.

\section{Acknowledgement}

We would like to sincerely thank the NGO SOSForêts (its President Dr. Wadja Mathieu Egnankou), all its partners (BirdLife International, CEPF) for their unfailing support and the material and financial assistance made available to us for this study. We would also like to sincerely thank the SODEFOR officials for granting access to the classified forest and for their logistical contribution to the realisation of this study. Our thanks go to Mr. Keh Oulaï, Water and Forestry Officer, and to the local population for their hospitality and sympathy during their stay in the field.

\section{References}

Ahon, D. B., Kouassi, K. L., Zean, G. M. Béné, J-C K. \& Tano, Y. 2020. Inventaires préliminaires des oiseaux de la Réserve de Flore et de Faune du Haut Bandama, Centre-Nord, Côte d'Ivoire. Afrique SCIENCE, 17 (4) : 48 - 62

Bennun, L., Davies, G., Howell, K., Newing, H. Linkie, M., 2004. La biodiversité des forêts d'Afrique : Manuel pratique de recensement des vertébrés. Earthwatch Institute (Europe): Royaume-Uni; 2, 1-186.

Bibby, C. J, Burgess, N. D., Hill, D. A., 1992. Bird Census Techniques. Academic Press, London, England. 257pp.

Bibby, C., Burgess, N., Hill, D. Mustoe, S., 2000. Bird Census Techniques. (Academic Press: San Diego, CA.).

Bitty, A. E., Kadjo, B., Gonedele bi, O. O. M., Kouassi, K. K., 2013. Inventaire de la faune mammalogique d'une forêt urbaine, le Parc National du Banco, Côte d'Ivoire. Int. J. Biol. Chem. Sci. 7 (4), 1678-1687, 10 .

Borrow, N., Demey, R., 2001. Field Guide to the Birds of Western Africa. Christopher Helm: London, England, UK; 832 p.

Brou, Y. T., Oswald, Y., Bigot, S. Servat, E., 2005. Risque de déforestation dans le domaine 
permanent de l'Etat en Côte d'Ivoire : quel avenir pour ses derniers massifs forestiers ? TELA, Revue de télédetection de l'UAF, 5 (1-23), 17-33.

Chappuis, C., 2000. Oiseaux d'Afrique. 15 CDs. Paris, France. Société d'étude Ornithologique.

Delahaye, L., 2006. Sélection et modélisation de l'habitat d'oiseaux en chênaies et hêtraies ardennaises : étude de l'impact de la composition et de la structure forestière. Thèse de doctorat. Université de Gembloux, $401 \mathrm{p}$.

Demey, R., Rainey, H., 2005. Inventaire rapide des oiseaux des forêts classées de la Haute Dodo et du Cavally. Une Evaluation Biologique de Deux Forêts Classées du Sud-Ouest de la Côte d'Ivoire. Bulletin RAP d'Evaluation Rapide 34, Conservation International : Washington, D.C. : 76- 83 .

Eldin, M., 1971. Le climat de la Côte d'Ivoire. In: Le milieu naturel de la Côte d'Ivoire. Mémoire ORSTOM, 5O, Paris (France): 73-108.

Fishpool, L. D. C., Evans, M. I., 2001. Important Bird Areas in Africa and Associated Islands. BirdLife International, Cambridge, Grande Bretagne, 27p.

Gottschalk, T. K., Ekschmitt, K. Bairlein, \$ F., 2007. Relationships between vegetation and bird community composition in grasslands of the Serengeti. Afri. J Ecol. 45 (4), 557-565.

Guillaumet, J. L., Adjanohoun, E., 1971. La végétation de la Cote d'Ivoire. In : Le milieu naturel de Côte d'Ivoire. Mémoires ORSTOM, 50, Paris (France), pp 161- 263.

Halle, B., Bruzon, V., 2006. Profil Environnemental de la Cote d'Ivoire. Rapport final, AGRIFOR Consult, Belgique, 128 p.

Issiaka, Y., 2002. Indicateurs des avantages potentiels d'une zone humide : cas de la plaine d'inondation de N'Dounga-Sebéry (Niger). Mémoire de DEA. Université d'Abobo-Adjamé (RCI), $53 \mathrm{p}$.

Issiaka, Y., 2011. Importances des zones humides du parc National du $\mathrm{W}$ du Niger pour les oiseaux d'eau Afro-tropicaux et migrateurs du Paléarctique Occidental. Thèse de Doctorat. Université Abdou Moumouni (Niger), 149 p.

Konan, K. M., 2009. Diversité morphologique et génétique des crevettes des genres Atya Leach, 1816 et Macrobrachium Bate, 1868 de Côte d'Ivoire. Thèse de Doctorat, URF des Sciences et Gestion de l'Environnement, Université AboboAdjamé (Abidjan, Côte d'Ivoire), 189 p.
Koné, M., Kouadio, Y. L., Neuba, D. F. R., Malan, D. F., Coulibaly, L., 2014. Evolution de la couverture forestière de la Côte d'Ivoire des années 1960 au début du 21e siècle. Int. J Innov. and App. Stu., 7 (2), 782-794.

Kouadio, K. P., Yaokokoré-béibro, K. H., Odoukpe, K. S. G., Konan, E. M., N'guessan, A. M. Kouassi, K. P., 2014. Diversité avifaunique de la forêt classée de N'ganda N'ganda (Sud-Est de la Côte d'Ivoire). Afri. Sci., 10 (1), 1-13

Kouakou, A. T. M., Barima, Y. S. S., Kouakou, K. A., Kouamé, N. F., Bogaert, J., Kouadio, Y. J., 2015. Forest Dynamics in the North of the Classified Forest of Haut-Sassandra During the Period of Armed Conflicts in Ivory Coast. Ameri. J Life Sci., 3 (5), 375-382.

Lepage, D., 2017. Liste des oiseaux de Côte d'Ivoire. Avibase, la base de données mondiale des oiseaux. Consulté sur https : //avibase.bsceoc.org, le [24/09/2020].

Lévêque, C., 1994. Environnement et diversité du vivant. Collection Explora ; 127 p.

Lougbégnon, O., Codjia, T. J. T. C., Libois M. R., 2007. Biodiversité et distribution écologique de l'avifaune des plantations du Bénin. Actes du $1^{\mathrm{er}}$ colloque de l'UAC des Sciences Cultures et Technologies, Agronomie, p 47-67

Murhabale, B. C., Irenge, B. C., Biringanine, G. K., Bapeamoni, F. A., Kahindo C. M., Upoki, D. A. 2020. Evaluation des connaissances et de l'impact des pratiques des populations riveraines sur la conservation de l'avifaune de la Forêt de Burhinyi (Itombwe, Sud-Kivu, RD Congo). International Journal of Biological and Chemical Sciences, 14 (6) : 1999-2017.

Mayaux, P., Bartholomé, E., Massart, M., Van Cutsem, C., Cabral, A., Nonguierma, A., Diallo, O., Pretorius, C., Thompson, M., Cherlet, M., Pekel, J-F., Defourny, P., Vasconcelos, M., Di Gregorio, A., Fritz, S., De Grandi, G., Elvidge, C., Vogt, P., Belward, A., 2003. Cartedu l'occupation de l'Afrique, EUR 20665 EN, European Commission, Joint Research Center, $56 \mathrm{p}$.

Meffe, G. K., Caroll, C. R., 1994. Principes of conservation biology. Sinauer Associates Inc., Sunderland, Massachuettes.

Monnier, Y., 1983. Carte de la végétation de la Côte-d'Ivoire. In : Vennetier P \& Laclavère G. (eds), Atlas de la Côte d'Ivoire. 2e édition, 72 pp., Paris: Jeune Afrique. 
N’Da, D. H., N'Guessan, K. E., Wadja, E. M., Kouadio, A., 2008. Apport de la télédétection au suivi de la déforestation dans le parc national de la Marahoué (Côte d'Ivoire). Télédétection, 8 (1), $17-34$.

Puig, H. 2001. Diversité Spécifique et déforestation : l'exemple des forêts tropicales humides du Mexique. Bois et Forêts des Tropiques, 268 (2), 41-55.

Saunders, D. A., Hobbs, R., Margules, C. R., 1991. Biological consequences of ecosystem fragmentation. Conserv. Biol., 5, 18-32.

Stattersfield, A. J., Crosby, M. J., Long, A. J., Wege, D. C., 1998. Endemic Bird Areas of the World: Priorities for Biodiversity Conservation. BirdLife International. Cambridge, UK. Series No. 7, 846 p.

Teyssèdre, A., 2004. Vers une sixième grande crise d'extinctions ? In : Barbault R. \& Chevassus-auLouis B., eds. Biodiversité et changements globaux. Enjeux de société et défis pour la recherche. Paris : Association pour la Diffusion de la Pensée Française (ADPF), 24-36.

IUCN, 2020. Liste rouge l'Union Internationale pour la Conservation de la Nature www.iucn.org, consulté 31/o8/2020.

Vallan, D., 1999. Consequences of degradation and fragmentation of the Malagasy rainforest on amphibian communities. Bem.

Yaokokoré-Béibro, K. H., 2001. Avifaune des forêts classées de l'Est de la Côte d'Ivoire : données sur l'écologie des espèces et effet de la déforestation sur les peuplements. Cas des forêts classées de la Béki et de la Bossématié (Abengourou). Thèse de Doctorat de l'Université de Cocody, 245 p.

Yaokokoré-Béibro, K. H., 2010. Diversité avifaunique de la forêt classée de la Besso, SudEst de la Côte d'Ivoire. Sciences \& Nature, 7 (2) : $207-219$

Yaokokoré-Béibro, K. H., Konan, E. M. Kouadio, K. P., 2015. Diversité et abondance des oiseaux de la forêt classée de la Téné, Centre-Ouest Côte d'Ivoire. J Ani. \& Sci., 24: 1-11.

Zean, G. M., Ahon, D. B., Béné, J-C. K., 2018. Peuplement avifaunique du Campus Universitaire Jean Lorougnon Guédé, Daloa et sa périphérie (Centre-Ouest de la Côte d'Ivoire). Int. J. Biol. Chem. Sci. 12 (6), 25032518.

\section{How to cite this article:}

Zean, G. M., Ahon, D. B., Koffi, B. J., 2020. Birds diversity of the Bouafle Classified Forest (Centralwestern of Côte d'Ivoire). Int. J. Curr. Res. Biosci. Plant Biol. 7(11), 1-13.

doi: https://doi.org/10.20546/ijcrbp.2020.711.001 